\title{
Development of a Spectral Camera for Cartilage Monitoring
}

\author{
A. Kuehn, A.Graf, U. Wenzel, S. Princz, R. Miller, H. Mantz and M. Hessling \\ Ulm University of Applied Sciences, Department of Mechatronics and Medical Engineering \\ Albert-Einstein-Allee 55, D-89081 UIm (Germany), hessling@hs-ulm.de
}

\begin{abstract}
:
In a joint research project with the UIm University Medical Center, the UIm University of Applied Sciences is developing a bioreactor for tissue engineering of facial cartilage. The cartilage growth will be monitored by a spectral camera to obtain cartilage composition and thereby to ensure cartilage quality. By excitation the cartilage with UV light, it is possible to record low intensity fluorescence spectra in the spectral range from 380 to $500 \mathrm{~nm}$ over the surface of a cartilage specimen. At present the camera records fluorescence spectra along a line of several hundred points. Further developments will allow obtaining fluorescence spectra in two dimensions by a single snapshot.
\end{abstract}

Key words: Spectral camera, spectrometry, facial cartilage, tissue engineering

\section{Introduction}

Facial cartilage defects caused by diseases or accidents can lead to grave psychological and physiological consequences for the patient. Defects should ideally be replaced with autologous cartilage material and tissue engineering is an appropriate solution to produce this personalized implant from the patient's own cells [1, 2]. However, tissue engineering is a new research field with many experiments necessary on the path to suitable implants. The cartilage has to be monitored during the growth in the bioreactor to ensure a functioning cartilage tissue before it can be implanted in a patient. Present examination methods destroy the cartilage tissue so that it cannot be implanted. In order to overcome the problem of ruining cartilage tissue by examination, a reliable remote sensing system is needed to evaluate cartilage tissue before implantation and as well to check the state during the tissue growth. The most common fluorophores in the extracellular matrix are collagen and elastin. It is possible to distinguish collagen I \& II by their fluorescence response as well as elastin and glycosaminoglycans (GAG) [3, 4]. On this context one can make a point about the cartilage composition. One suitable and handy opportunity is to record these fluorescence with a spectral camera. Unfortunately the fluorescence radiation is very weak and the emission spectrum wavelength is between $380 \mathrm{~nm}$ and $500 \mathrm{~nm}$, but no commercial system is available in this range that has the sensitivity needed here. Another drawback of commercial systems are the high acquisition costs.

In this paper the development of a low cost and sensitive spectral camera for the short visible range will be presented, that is able to record fluorescence spectra of collagen I \& II and further cartilage compounds. It is based on an idea of Habel et al. [5]. First results are available.

\section{Cartilage fluorescence}

Cartilage is composed of a dense net of collagen and elastic fibers which are integrated in chondroitin sulfate which is a gel-like component of the ground substance. Fibers and ground substance form the extracellular matrix which surrounds the chondrocytes. Except for the perichondrium, cartilage doesn't contain blood vessels. Due to this fact cartilage grows very slowly and hardly heals after an injury [6]. The collagen molecules are stabilized by the intra- and intermolecular cross-links structure. Cross-links consist of pyridinoline which is responsible for the cartilage fluorescence [7, 8]. Increasing fluorescence intensity is associated with increased cross-links of collagen $[9,10]$.

\section{1-Dimensional Spectral Imaging}

The excitation of the cartilage sample is either performed by a $340 \mathrm{~nm}$ LED with $1.4 \mathrm{~mW}$ (EPIGAP Optronics, EOLD-340-TO) or a 365 nm LED with ca. 200 mW (LED Engin, LZ400U605). Because of a broad LED emission 


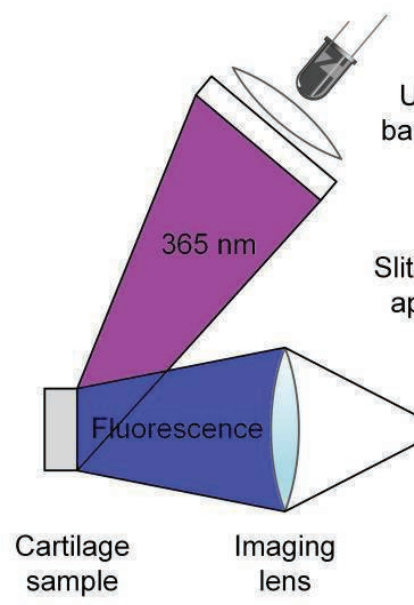

UV LED and

band-pass filter
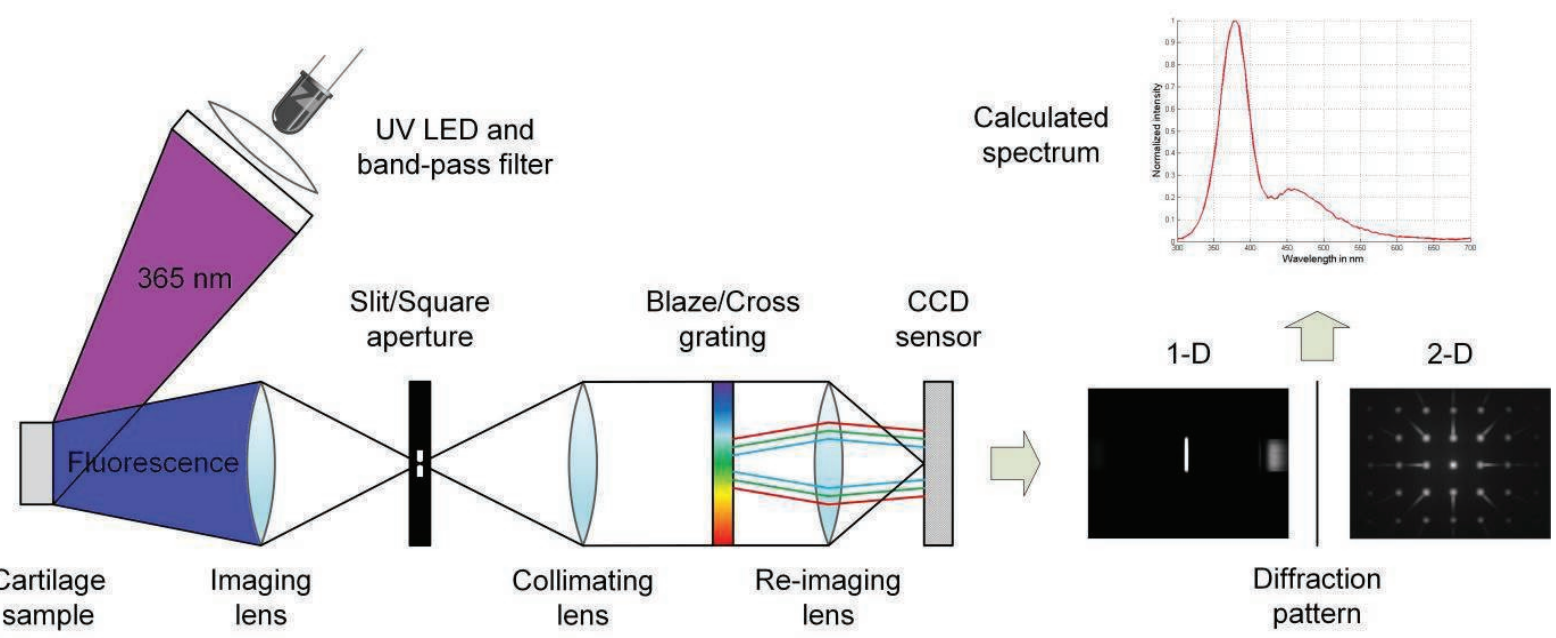

Fig. 1. Scheme of 1-/ 2-dimensional spectral camera setup and spectrum generation.

around $365 \mathrm{~nm}$ a band-pass filter (Delta, BP 365/12 (LF101653)) was used in combination with the $365 \mathrm{~nm}$ LED. A scene of the cartilage sample is imaged by a $35 \mathrm{~mm}$ lens onto a slit aperture. The slit width is $20 \mu \mathrm{m}$. After the slit aperture the light diverges and is collimated by a $25 \mathrm{~mm}$ lens. Subsequently a blazed line grating with $300 \mathrm{l} / \mathrm{mm}$ diffracts the light and a 30 $\mathrm{mm}$ re-imaging lens images it onto a monochrome CCD chip (Sony ICX -285 AL) with a resolution of $1392 \times 1040$ pixels (Fig. 1). The optical magnification is about 1.2 with a high numerical aperture of 0.3 . To reduce noise the CCD chip is cooled by a peltier element. From the captured pattern caused by the diffraction grating, a complete spectrum can be calculated for every position of the slit, respectively, for every point of the line sample (Fig. 2). In a range from 360 to $510 \mathrm{~nm}$ it is possible to determine 550 fluorescence spectra with a spectral resolution of 2 to $3 \mathrm{~nm}$ [11].

\section{2-Dimensional Spectral Imaging}

Recording spectra over a line is an improvement compared to measuring a spectrum of a single point. The cartilage implant will reach a size of several square centimeters. For that reason a 2-dimensional system is required for analyzing the whole cartilage sample at once. The setup of the 2-dimensional optical system is similar to the 1-dimensional one (Fig. 1). Instead of a slit aperture a square aperture is used which is made of razor blades. The blazed line grating is replaced by a transmission cross grating with $200 \mathrm{l} / \mathrm{mm}$ which produces a more complex diffraction pattern (see Fig. 3). The reimaging lens was replaced by a $16 \mathrm{~mm}$ lens to capture the whole diffraction pattern. This method allows to record spectra in a single snapshot. The so-called computed tomography imaging spectrometer (CTIS) is based on the principles used for computed tomography. The diffraction pattern can be considered as parallel projections of voxels of a 3-d object cube. Two dimensions are the spatial coordinates and one dimension is the wavelength [12]. The voxels of the object cube can be denoted as a long serialized vector $\vec{f}$. The effect of the imaging system can be described by a matrix $H$ with the size of $M \times N$, where $\mathrm{N}$ is the number of voxels in the object cube and $\mathrm{N}$ is the number of pixels in the image. Operating the system matrix $H$ on the object cube vector $\vec{f}$ produces the vector $\vec{g}$,

$$
\vec{g}=H \vec{f}
$$

which is representing the serialized pixels in the image. From these projections one can reconstruct the 3-d object cube with the Expectation Maximization algorithm,

$\hat{f}_{n}^{k+1}=\frac{\hat{f}_{n}^{k}}{\sum_{m=1}^{M} H_{m n}} \sum_{m=1}^{M} H_{n m}^{T} \frac{g_{m}}{\left(H \hat{f}^{k}\right)_{m}}$

with the first guess as $\overrightarrow{\hat{f}}^{0}=H^{T} \vec{g}$. In this case the system converges after 10 iterations [13]. Thus it is possible to obtain a spectrum for each point of the square aperture (Fig. 5). A theoretical spectral resolution of approximately $4 \mathrm{~nm}$ can be achieved. The algorithms were implemented and calculated with MATLAB R2013a. Currently it takes 7 seconds to calculate the spectrum for one point.

As sample processed collagen I \& II were used. The spectra for comparison were acquired with a spectrometer (Avantes, SensLine AvaSpec2048 XL) [4]. 

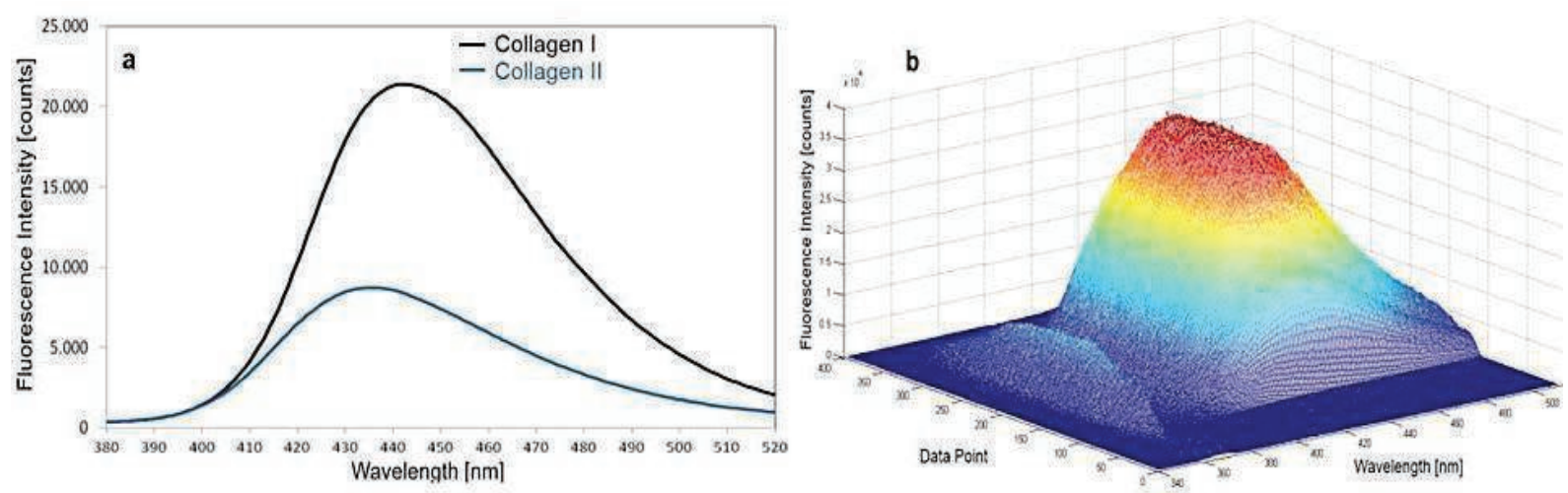

Fig. 2. (a) Single fluorescence spectra for collagen I \& II with $365 \mathrm{~nm}$ excitation. (b) Several hundred simultaneously recorded fluorescence spectra of collagen I with $365 \mathrm{~nm}$ excitation [11].

\section{Results}

Fig. 2 (a) shows the fluorescence spectrum of a single point of the slit and a complete dataset along the slit (b). The excitation was carried out with the $365 \mathrm{~nm}$ LED with an integration time of $10 \mathrm{~s}$ for collagen I and with an integration time of $60 \mathrm{~s}$ for collagen II. One can see a maximum in the range of 440 to $445 \mathrm{~nm}$ for collagen I and a maximum in the range of 432 to $437 \mathrm{~nm}$ for collagen II. It's visible that the fluorescence intensity of collagen I is much higher than that of collagen II, especially when regarding the integration time. Fig. 2 (b) presents a dataset of fluorescence spectra of collagen I along a line with 300 data points with an excitation of $365 \mathrm{~nm}$. The first small peak area at $365 \mathrm{~nm}$ is the reflected excitation light.

The diffraction pattern of the 2-dimensional system can be seen in Fig. 3 for collagen I fluorescence. A long integration time was chosen to obtain a good signal. The spatial expansion of the square aperture on the CCD chip is $17 \times 14$ pixels. The high intensity "dots" are the reflections of the excitation LED (365 $\mathrm{nm})$. The blurred lines are the detected fluorescence. One can notice that the diffraction patterns are blurry. This could be caused by chromatic aberration of the employed optical lenses in the UV range. Fig. 4 presents the spectrum of one point in Fig. 3 (red line). Before calculating, the dark signal was subtracted. The intensity is represented normalized. For comparison a spectrum is presented which was measured with a spectrometer (black line) [4]. The first peak at $365 \mathrm{~nm}$ is again the reflected excitation light. It is clearly visible that the width of the spectrum produced by the camera is higher. This can be explained by a lower spectral resolution compared to the spectrometer. Nevertheless one can see a fluorescence maximum in the range of 440 to
$450 \mathrm{~nm}$. It is most closely corresponding to the spectrum acquired with the 1-dimensional system as well as to spectrometer data. Fig. 5 shows a dataset for all points of the square aperture, respectively of the sample.

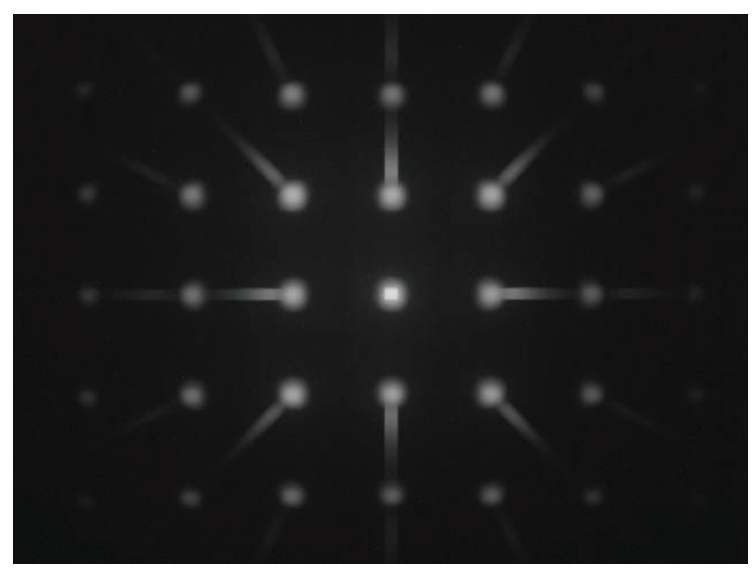

Fig. 3. 2-dimensional diffraction pattern of collagen I fluorescence. Excited with $365 \mathrm{~nm}$ for $180 \mathrm{~s}$.

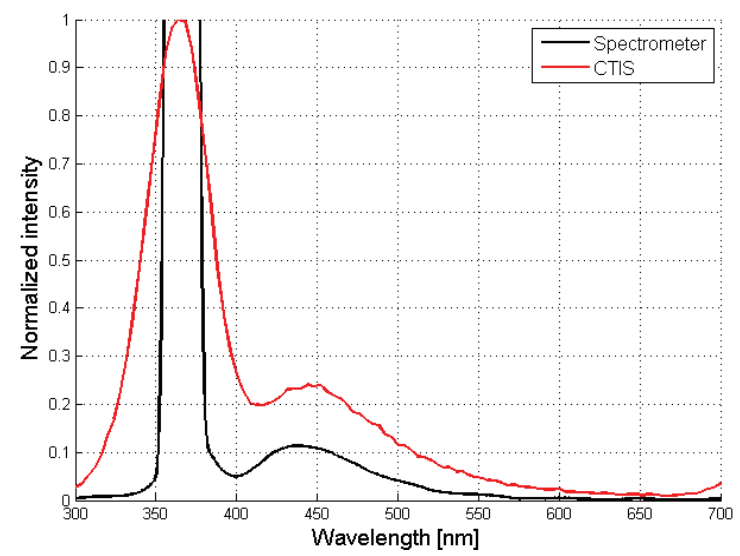

Fig. 4. Fluorescence spectrum of collagen I of a single point, acquired with the CTIS (red). Excited with $365 \mathrm{~nm}$ for $180 \mathrm{~s}$. Fluorescence spectrum of collagen I of a single point, acquired with a spectrometer and amplified with 10 (black). Excited with $365 \mathrm{~nm}$ with an integration time of $0.05 \mathrm{~s}$ [4]. 


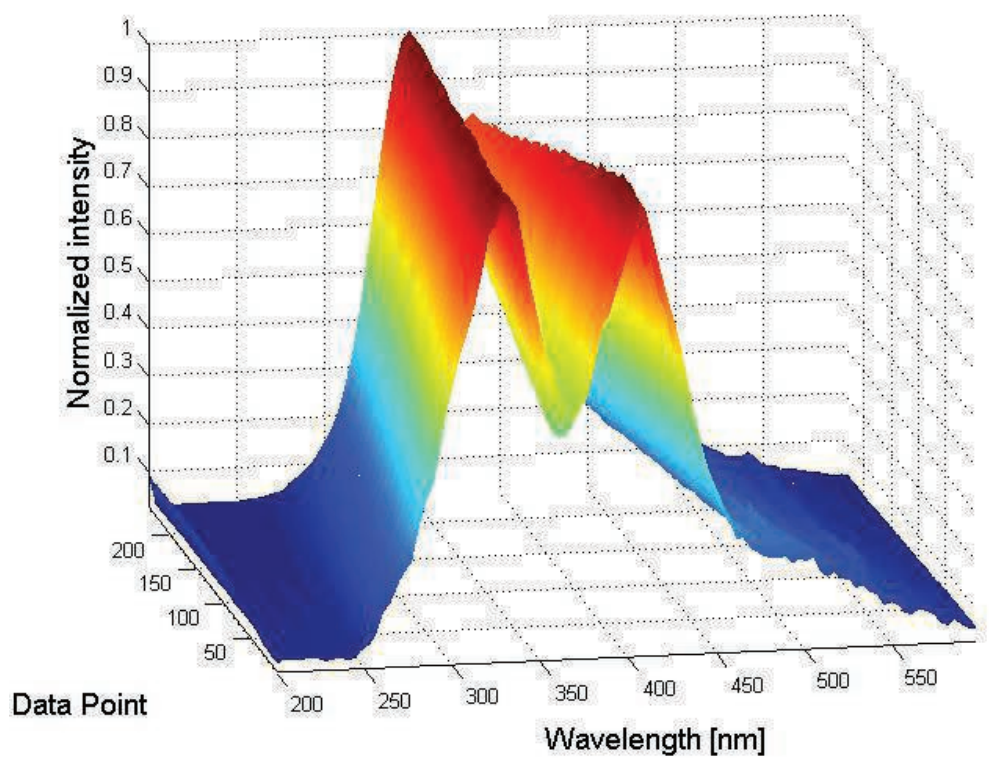

Fig. 5. Spectra of all 238 data points of Fig. 3.

\section{Discussion and conclusion}

It was shown that it is possible to distinguish collagen | from collagen II by their spectral response with the 1-dimensional spectral imaging. The obtained spectra are highly resolved.

The 2-dimensional spectral imaging delivers very promising results but needs to be improved in terms of spectral resolution. One approach could be to filter noise. In this case noise was treated as a signal. This could be a reason for the lower resolution of the 2dimensional system, since with each iteration of the reconstruction algorithm, the noise is amplified [14]. Another approach for increasing the resolution can be achieved by decreasing the grating constant of the 2-d grating. This would increase the dispersion and thereby the signal overlapping wouldn't be that distinctive. Replacing the current lenses with specialized lenses which operate in the UV range would also improve the spectral resolution. The first measurements were carried out with an aperture which takes $17 \times 14$ pixels. Considering the interested range of 380 to $500 \mathrm{~nm}$, the aperture can be increased to cover $100 \times 100$ pixels of which one can obtain a spectrum for every point. This will also lead to a more smeared diffraction image that will also impair the spectra accuracy.

After optimization the system measurements for elastin and GAG are planned.

\section{Acknowledgement}

Support of the "Bundesministerium für Bildung und Forschung" (Förderkennzeichen
03FH008I3), the UIm University Medical Center (Prof. Rotter and Dr. Schwarz of the Department of Oto-Rhino-Laryngology Head and Neck Surgery) and the Institut für Lasertechnologien in der Medizin und Messtechnik UIm (Dr. Scalfi-Happ and Dr. Stock) is gratefully acknowledged!

\section{References}

[1] N. Rotter, M. Bücheler, A. Haisch, B. Wollenberg, and S. Lang, "Cartilage tissue engineering using resorbable scaffolds," (eng), Journal of tissue engineering and regenerative medicine, vol. 1 , no. 6 , pp. 411416, 2007.

[2] U. R. Gössler and K. Hörmann, "Neue Strategien zum Gewebeersatz im Kopf-HalsBereich," (ger), HNO, vol. 57, no. 2, pp. 100$112,2009$.

[3] V.-D. Tuan and M. C. Brian, "Fluorescence spectroscopy for biomedical diagnostics," in Biomedical Photonics Handbook, T. Vo-Dinh, Ed, Florida: CRC Press, 2003, pp. 1-51.

[4] M. Zoller, "Nichtinvasive auf Fluoreszenz basierende punktuelle Knorpelbeurteilung für einen Tissue-Engineering-Bioreaktor,"

Master-Thesis, Hochschule Ulm, Ulm, 2014.

[5] R. Habel, M. Kudenov, and M. Wimmer, "Practical Spectral Photography," Computer Graphics Forum, vol. 31, no. 2pt2, pp. 449458, 2012.

[6] G. J. Tortora and B. H. Derrickson, Anatomie und Physiologie. Weinheim: Wiley- $\mathrm{VCH}$, 2006.

[7] D. Fujimoto, K.-y. Akiba, and N. Nakamura, "Isolation and characterization of a fluorescent material in bovine achilles tendon collagen," Biochemical and Biophysical Research Communications, vol. 76, no. 4, pp. 11241129 , 
http://www.sciencedirect.com/science/article/p ii/0006291X7790972X, 1977.

[8] A. Uchiyama, T. Inoue, and D. Fujimoto, "Synthesis of pyridinoline during in vitro aging of bone collagen," (eng), J Biochem, vol. 90, no. 6, pp. 1795-1798, 1981.

[9] K. Sokolov, J. Galvan, A. Myakov, A. Lacy, R. Lotan, and R. Richards-Kortum, "Realistic three-dimensional epithelial tissue phantoms for biomedical optics," (eng), Journal of biomedical optics, vol. 7 , no. 1, pp. 148-156, 2002.

[10] N. D. Kirkpatrick, J. B. Hoying, S. K. Botting, J. A. Weiss, and U. Utzinger, "In vitro model for endogenous optical signatures of collagen," (eng), Journal of biomedical optics, vol. 11, no. 5, p. 054021, 2006.

[11] A. Graf, "Aufbau einer Spektralkamera zur Fluoreszenzanalyse von Knorpelgewebe," Bachelor-Thesis, Hochschule UIm, UIm, 2014.

[12] T. Okamoto and I. Yamaguchi, "Simultaneous acquisition of spectral image information," Opt. Lett, vol. 16, no. 16, p. 1277, 1991.

[13] M.R. Descour and E.L. Dereniak, Eds, Nonscanning no-moving-parts imaging spectrometer: International Society for Optics and Photonics, 1995.

[14] N. Hagen, E. L. Dereniak, D. T. Sass, S. S. Shen, and P. E. Lewis, "Maximizing the resolution of a CTIS instrument," in Optics \& Photonics: SPIE, 2006, pp. 63020L. 